\title{
Self-Adjoint Extensions with Friedrichs Lower Bound
}

\author{
Matteo Gallone ${ }^{1}$ (D) Alessandro Michelangeli ${ }^{2}$
}

Received: 28 April 2020 / Accepted: 29 August 2020 / Published online: 11 September 2020

(c) The Author(s) 2020

\begin{abstract}
We produce a simple criterion and a constructive recipe to identify those self-adjoint extensions of a lower semi-bounded symmetric operator on Hilbert space which have the same lower bound as the Friedrichs extension. Applications of this abstract result to a few instructive examples are then discussed.
\end{abstract}

Keywords Lower semi-bounded symmetric operators - Self-adjoint extensions · Friedrichs extensions

Mathematics Subject Classification 47B15 $\cdot 47 \mathrm{~B} 25 \cdot 47 \mathrm{~N} 50 \cdot 81 \mathrm{Q} 10$

\section{Motivation}

We start with a familiar example. In the Hilbert space $\mathcal{H}=L^{2}(0,1)$ let us consider the densely defined, closed, and symmetric operator

Communicated by Daniel Aron Alpay.

Partially supported by the Istituto Nazionale di Alta Matematica (INdAM) and the Alexander von Humboldt foundation.

This article is part of the topical collection "Spectral Theory and Operators in Mathematical Physics" edited by Jussi Behrndt, Fabrizio Colombo and Sergey Naboko.

Matteo Gallone

matteo.gallone@unimi.it

Alessandro Michelangeli

michelangeli@iam.uni-bonn.de

1 Dipartimento di Matematica, Università degli Studi di Milano, via Cesare Saldini 50, 20133

Milan, Italy

2 Institute for Applied Mathematics, and Hausdorff Center for Mathematics, University of Bonn, Endenicher Allee 60, 53115 Bonn, Germany 


$$
S=-\frac{\mathrm{d}^{2}}{\mathrm{~d} x^{2}}, \quad \mathcal{D}(S)=H_{0}^{2}(0,1)=\left\{\begin{array}{l|l}
f \in H^{2}(0,1) & \begin{array}{c}
f(0)=0=f(1) \\
f^{\prime}(0)=0=f^{\prime}(1)
\end{array}
\end{array}\right\}
$$

$S$ is actually the operator closure of the negative Laplacian defined on $C_{0}^{\infty}(0,1)$. Here and in the following $\mathcal{D}(R)$ denotes the domain of the operator $R$ acting on $\mathcal{H}$, and if $R$ is symmetric we denote by

$$
\mathfrak{m}(R):=\inf _{\substack{f \in \mathcal{D}(R) \\ f \neq 0}} \frac{\langle f, R f\rangle}{\|f\|_{2}^{2}} \in[-\infty,+\infty)
$$

the largest lower bound of $R$. When $\mathfrak{m}(R)>-\infty$ one says that $R$ is semi-bounded from below. Poincaré inequality implies that $S$ is semi-bounded from below with

$$
\mathfrak{m}(S)=\pi^{2} .
$$

Now, $S$ is symmetric but not self-adjoint, for

$$
S^{*}=-\frac{\mathrm{d}^{2}}{\mathrm{~d} x^{2}}, \quad \mathcal{D}\left(S^{*}\right)=H^{2}(0,1) .
$$

Thus, $S$ admits a multiplicity (in fact, a four-real-parameter family) of distinct selfadjoint extensions, which are all restrictions of $S^{*}$. Among them, the Friedrichs extension $S_{F}$ is the one with domain

$$
\mathcal{D}\left(S_{F}\right)=H^{2}(0,1) \cap H_{0}^{1}(0,1)=\left\{f \in H^{2}(0,1) \mid f(0)=0=f(1)\right\},
$$

namely the Dirichlet (negative) Laplacian. Let us recall that abstractly speaking the Friedrichs extension of a lower semi-bounded symmetric operator $S$ is the only selfadjoint extension of $S$ with the property

$$
\mathcal{D}\left(S_{F}\right) \subset \mathcal{D}[S]
$$

that is, with operator domain contained in the form domain of $S$. Here and in the following $\mathcal{D}[R]$ denotes the form domain of a lower semi-bounded symmetric operator $R$, or also of a self-adjoint operator $R$ (see, e.g., [11, Chapt. 10]); in the present case

$$
\mathcal{D}[S]=\overline{\mathcal{D}}(S)^{\|\|_{H^{1}}}=H_{0}^{1}(0,1)
$$

and obviously $\mathcal{D}[S]=\mathcal{D}\left[S_{F}\right]$. It is also a general property of the Friedrichs extension the fact that $S_{F} \geq \widetilde{S}$ for any other $\widetilde{S}=\widetilde{S}^{*} \supset S$, namely $S_{F}$ is the largest of all the self-adjoint extensions of $S$ in the sense of operator form ordering.

As well known, as follows from (1.5), $S_{F}$ is diagonalizable over an orthonormal basis $\{\sqrt{2} \sin n \pi x \mid n \in \mathbb{N}\}$ of eigenfunctions, with (simple and pure point) spectrum

$$
\sigma\left(S_{F}\right)=\left\{n^{2} \pi^{2} \mid n \in \mathbb{N}\right\}
$$


Thus,

$$
\mathfrak{m}\left(S_{F}\right)=\pi^{2}=\mathfrak{m}(S)
$$

which actually expresses a completely general fact: the Friedrichs extension of a lower semi-bounded operator preserves the lower bound. Whereas self-adjoint extensions of $S$ cannot increase the lower bound, in general they decrease it. For instance, in the example under consideration, the extension $S_{P}$ with periodic boundary conditions, namely with domain

$$
\mathcal{D}\left(S_{P}\right)=\left\{f \in H^{2}(0,1) \mid f(1)=f(0), f^{\prime}(1)=f^{\prime}(0)\right\},
$$

has spectrum

$$
\sigma\left(S_{P}\right)=\left\{n^{2} \pi^{2} \mid n \in \mathbb{N}_{0}\right\}, \quad \text { whence } \quad \mathfrak{m}\left(S_{P}\right)=0 .
$$

Yet, the extension $S_{A}$ with anti-periodic boundary conditions, namely with

$$
\mathcal{D}\left(S_{A}\right)=\left\{f \in H^{2}(0,1) \mid f(1)=-f(0), f^{\prime}(1)=-f^{\prime}(0)\right\},
$$

does preserve the lower bound of $S$. Indeed,

$$
\sigma\left(S_{A}\right)=\left\{(2 n+1)^{2} \pi^{2} \mid n \in \mathbb{N}_{0}\right\}, \quad \text { whence } \mathfrak{m}\left(S_{P}\right)=\pi^{2} .
$$

This occurrence is well known: a lower semi-bounded symmetric operator may admit self-adjoint extensions other than the Friedrichs, with the same bottom of the Friedrichs spectrum. Actually this is not typical of symmetric operators with deficiency index 2 only, as was the case for $S$ here. In Sect. 3 also examples with deficiency indices 1 will be recalled and discussed. By standard direct sum, these examples also cover the case of infinite deficiency indices.

Now, while the possibility of non-Friedrichs self-adjoint extensions with the same Friedrichs lower bound is folk knowledge, we are not aware of an explicit operatortheoretic explanation of this phenomenon, nor of a characterisation in terms of transparent conditions which, once they are met, allow to construct all extensions with such a feature.

In this note we present a simple criterion and a constructive recipe to identify those self-adjoint extensions of a lower semi-bounded symmetric operator on Hilbert space which have the same lower bound as the Friedrichs extension. The abstract main results, Theorems 2.4, 2.5, and 2.6 below, are discussed in Sect. 2, and illustrative concrete examples where such results can be applied to are then presented in Sect. 3.

\section{Abstract Results}

Let $\mathcal{H}$ be a Hilbert space (over $\mathbb{R}$ or $\mathbb{C}$, with scalar product $\langle\cdot, \cdot\rangle$ anti-linear in the first entry, and with norm \|\| ) and let $S$ be a densely defined, symmetric, semi-bounded 
operator on $\mathcal{H}$ with lower bound $\mathfrak{m}(S) . S$ in not necessarily closed. For clarity of the presentation we shall assume non-restrictively $\mathfrak{m}(S)>0$. This implies that $S_{F}^{-1}$ is everywhere defined and bounded on $\mathcal{H}$. It will be clear both from this abstract discussion and from the applications in Sect. 3 that the case of general finite $\mathfrak{m}(S)$ can be covered by suitably shifting $S$ to $S-\mu \mathbb{1}$ with $\mu<\mathfrak{m}(S)$.

Unless such $S$ is already essentially self-adjoint, it admits non-trivial self-adjoint extensions. In this case $\operatorname{ker} S^{*}$, the deficiency space for $S$, is non-trivial either. Standard extension schemes produce convenient classifications of the whole family of extensions. It can be shown within the modern theory of boundary triplets [2], or equivalently the classical 'universal' parametrization by Grubb [7], and in fact the very original extension theory by Krel̆n [9], Višik [12], and Birman [3], that the extensions of $S$ can be labelled as follows.

Theorem 2.1 There is a one-to-one correspondence between the family of all selfadjoint extensions of $S$ on $\mathcal{H}$ and the family of the self-adjoint operators on Hilbert subspaces of $\operatorname{ker} S^{*}$.

(i) If $T$ is any such operator, in the correspondence $T \leftrightarrow S_{T}$ each self-adjoint extension $S_{T}$ of $S$ is given by

$$
\begin{aligned}
S_{T} & =S^{*} \uparrow \mathcal{D}\left(S_{T}\right) \\
\mathcal{D}\left(S_{T}\right) & =\left\{\begin{array}{ll}
f+S_{F}^{-1}(T v+w)+v \mid \begin{array}{l}
f \in \mathcal{D}(\bar{S}), v \in \mathcal{D}(T) \\
w \in \operatorname{ker} S^{*} \cap \mathcal{D}(T)^{\perp}
\end{array}
\end{array}\right\} .
\end{aligned}
$$

(ii) If $S_{T}$ is a semi-bounded (not necessarily positive) self-adjoint extension of $S$, then

$$
\mathcal{D}[T]=\mathcal{D}\left[S_{T}\right] \cap \operatorname{ker} S^{*}
$$

and

$$
\begin{aligned}
\mathcal{D}\left[S_{T}\right]= & \mathcal{D}\left[S_{F}\right]+\mathcal{D}[T] \\
S_{T}\left[f+v, f^{\prime}+v^{\prime}\right]= & S_{F}\left[f, f^{\prime}\right]+T\left[v, v^{\prime}\right] \\
& \forall f, f^{\prime} \in \mathcal{D}\left[S_{F}\right], \forall v, v^{\prime} \in \mathcal{D}[T] .
\end{aligned}
$$

As a consequence,

$$
S_{T_{1}} \geq S_{T_{2}} \quad \Leftrightarrow \quad T_{1} \geq T_{2}
$$

(iii) If $\mathfrak{m}(T)>-\mathfrak{m}(S)$, then

$$
\mathfrak{m}(T) \geq \mathfrak{m}\left(S_{T}\right) \geq \frac{\mathfrak{m}(S) \mathfrak{m}(T)}{\mathfrak{m}(S)+\mathfrak{m}(T)}
$$

Theorem 2.1 collects results that are proved, e.g., in [8, Chapt. 13], [11, Chapt. 14], and [5, Sect. 3]. 
For convenience, let us denote by $\mathcal{S}(\mathcal{K})$ the collection of all self-adjoint operators defined in Hilbert subspaces of a given Hilbert space $\mathcal{K}$ : Theorem 2.1 states that the self-adjoint extensions of $S$ are all of the form $S_{T}$ for some $T \in \mathcal{S}\left(\operatorname{ker} S^{*}\right)$.

The Friedrichs extension of $S$ can be expressed in terms of the classical decomposition formula (see, e.g., [5, Sect. 2.2])

$$
\mathcal{D}\left(S_{F}\right)=\mathcal{D}(\bar{S}) \dot{+} S_{F}^{-1} \operatorname{ker} S^{*} .
$$

Therefore, $S_{F}$ is recovered from the general parametrisation (2.1) or (2.3) with the choice $\mathcal{D}[T]=\{0\}$ (thus, formally, “ $T=\infty$ ”).

An ancillary result that tends to be somehow less highlighted, but which is most relevant for our discussion, is the following.

Theorem 2.2 If, with respect to the notation of (2.1), $S_{T}$ is a self-adjoint extension of $S$, and if $\mu<\mathfrak{m}(S)$, then

$$
\begin{gathered}
\left\langle g, S_{T} g\right\rangle \geq \mu\|g\|^{2} \quad \forall g \in \mathcal{D}\left(S_{T}\right) \\
\text { 企 } \\
\langle v, T v\rangle \geq \mu\|v\|^{2}+\mu^{2}\left\langle v,\left(S_{F}-\mu \mathbb{1}\right)^{-1} v\right\rangle \quad \forall v \in \mathcal{D}(T) .
\end{gathered}
$$

As an immediate consequence, Theorem 2.2 reproduces the inequality $\mathfrak{m}(T) \geq$ $\mathfrak{m}\left(S_{T}\right)$ for any semi-bounded $S_{T}$ and shows, in particular, that positivity or strict positivity of the bottom of $S_{T}$ is equivalent to the same property for $T$, that is,

$$
\begin{aligned}
& \mathfrak{m}\left(S_{T}\right) \geq 0 \quad \Leftrightarrow \quad \mathfrak{m}(T) \geq 0 \\
& \mathfrak{m}\left(S_{T}\right)>0 \quad \Leftrightarrow \quad \mathfrak{m}(T)>0 .
\end{aligned}
$$

To make this presentation self-contained, and for later convenience, let us deduce Theorem 2.2 from Theorem 2.1. To this aim, let us first single out a simple operatortheoretic property.

Lemma 2.3 If $A$ is a self-adjoint operator on a Hilbert space $\mathcal{H}$ with positive bottom $(\mathfrak{m}(A)>0)$, then

$$
\sup _{f \in \mathcal{D}(A)} \frac{|\langle f, h\rangle|^{2}}{\langle f, A f\rangle}=\left\langle h, A^{-1} h\right\rangle \quad \forall h \in \mathcal{H} .
$$

Proof Setting $g:=A^{1 / 2} f$ one has

$$
\sup _{f \in \mathcal{D}(A)} \frac{|\langle f, h\rangle|^{2}}{\langle f, A f\rangle}=\sup _{g \in \mathcal{H}} \frac{\left|\left\langle A^{-1 / 2} g, h\right\rangle\right|^{2}}{\|g\|^{2}}=\sup _{\|g\|=1}\left|\left\langle g, A^{-1 / 2} h\right\rangle\right|^{2}
$$

and since $\left|\left\langle g, A^{-1 / 2} h\right\rangle\right|$ attains its maximum for $g=A^{-1 / 2} h /\left\|A^{-1 / 2} h\right\|$, the conclusion then follows. 
Proof of Theorem 2.2 For generic $f \in \mathcal{D}\left(S_{F}\right)$ and $v \in \mathcal{D}(T)$, one has $g:=f+v \in$ $\mathcal{D}\left(S_{T}\right)$ and

$$
S_{T}[g]=\left\langle f, S_{F} f\right\rangle+\langle v, T v\rangle .
$$

Thus, $S_{T} \geq \mu \mathbb{1}$ is tantamount as requiring for all such $g$ 's that

$$
\left\langle f, S_{F} f\right\rangle+\langle v, T v\rangle \geq \mu(\langle f, f\rangle+\langle f, v\rangle+\langle v, f\rangle+\langle v, v\rangle)
$$

whence also, replacing $f \mapsto \lambda f, v \mapsto \gamma v$,

$$
\begin{aligned}
& \left(\left\langle f, S_{F} f\right\rangle-\mu\|f\|^{2}\right)|\lambda|^{2}-\mu\langle f, v\rangle \bar{\lambda} \gamma-\mu\langle v, f\rangle \lambda \bar{\gamma} \\
& +\left(\langle v, T v\rangle-\mu\|v\|^{2}\right)|\gamma|^{2} \geq 0 \quad \forall \lambda, \gamma \in \mathbb{C} .
\end{aligned}
$$

Since $\mu<\mathfrak{m}(S)$, and hence $\left\langle f, S_{F} f\right\rangle-\mu\|f\|^{2}>0$, last inequality holds true if and only if

$$
\mu^{2}|\langle f, v\rangle|^{2} \leq\left(\langle v, T v\rangle-\mu\|v\|^{2}\right)\left(\left\langle f, S_{F} f\right\rangle-\mu\|f\|^{2}\right)
$$

for arbitrary $f \in \mathcal{D}\left(S_{F}\right)$ and $v \in \mathcal{D}(T)$. By re-writing (*) as

$$
\langle v, T v\rangle-\mu\|v\|^{2} \geq \mu^{2} \frac{|\langle f, v\rangle|^{2}}{\left\langle f,\left(S_{F}-\mu \mathbb{1}\right) f\right\rangle}
$$

and by the fact that the above inequality is valid for arbitrary $f \in \mathcal{D}\left(S_{F}\right)$ and hence holds true also when the supremum over such $f$ 's is taken, Lemma 2.3 then yields

$$
\langle v, T v\rangle-\mu\|v\|^{2} \geq \mu^{2}\left\langle v,\left(S_{F}-\mu \mathbb{1}\right)^{-1} v\right\rangle,
$$

which completes the proof.

With these abstract results at hand, let us now turn to the identification of the non-Friedrichs extensions with the same Friedrichs lower bound.

It is worth observing that inequality (2.5) is not informative in this respect: indeed, owing to (2.5), a sufficient condition for the bottom of $S_{T}$ to equal the bottom of $S_{F}$ would be to impose $\mathfrak{m}(S) \mathfrak{m}(T) /(\mathfrak{m}(S)+\mathfrak{m}(T)) \geq \mathfrak{m}(S)$, but such inequality is only satisfied, in the form of an identity, when $\mathfrak{m}(T)=\infty$, therefore the above sufficient condition only selects $S_{T}=S_{F}$, the Friedrichs extension.

We rather focus on (2.7) from Theorem 2.2. There, the operator $S_{F}-\mu \mathbb{1}$ is invertible with everywhere bounded inverse on the whole $\mathcal{H}$ : indeed, $\mu<\mathfrak{m}(S)$ and then $\mathfrak{m}\left(S_{F}-\right.$ $\mu \mathbb{1})>0$. Instead, $S_{F}-\mathfrak{m}(S) \mathbb{1}$ fails to be invertible on $\mathcal{H}$, because its bottom is by construction equal to zero.

The informal idea now is that even if $\left(S_{F}-\mathfrak{m}(S) \mathbb{1}\right)^{-1}$ cannot be defined as a bounded operator on the whole $\mathcal{H}$, yet it makes sense on $\operatorname{ran}\left(S_{F}-\mathfrak{m}(S) \mathbb{1}\right)$, and if it happens that the latter space has a non-trivial intersection with $\operatorname{ker} S^{*}$, then there are 
non-zero vectors $v \in \operatorname{ran}\left(S_{F}-\mathfrak{m}(S) \mathbb{1}\right) \cap \operatorname{ker} S^{*}$ on which $\left\langle v,\left(S_{F}-\mathfrak{m}(S) \mathbb{1}\right)^{-1} v\right\rangle$ is unambiguously defined and hence the right-hand side of the second expression in (2.7) is meaningful also when $\mu=\mathfrak{m}(S)$. Moreover, if on such $v$ 's one can define an operator $T \in \mathcal{S}\left(\operatorname{ker} S^{*}\right)$ satisfying (2.7) when $\mu=\mathfrak{m}(S)$, then by suitably exploiting the limit $\mu \uparrow \mathfrak{m}(S)$ this should give a characterisation of the condition $\mathfrak{m}\left(S_{T}\right) \geq \mathfrak{m}(S)$, which is equivalent to $\mathfrak{m}\left(S_{T}\right)=\mathfrak{m}(S)$, as $S_{F} \geq S_{T}$, the Friedrichs extension is the largest of all self-adjoint extensions of $S$.

By elaborating on such idea we finally come to our main results, Theorems 2.4, 2.5, and 2.6 below.

Clearly, underlying (2.7) is the quadratic form language, so the actual operator to possibly invert in some subspace of $\operatorname{ker} S^{*}$ is rather $\left(S_{F}-\mathfrak{m}(S) \mathbb{1}\right)^{1 / 2}$, a positive self-adjoint operator with zero lower bound.

In this respect, as $S_{F}$ is self-adjoint on $\mathcal{H}$, and so is $S_{F}-\mathfrak{m}(S) \mathbb{1}$ with lower bound zero, then upon decomposing

$$
\mathcal{H}=\overline{\operatorname{ran}\left(S_{F}-\mathfrak{m}(S) \mathbb{1}\right)} \oplus \operatorname{ker}\left(S_{F}-\mathfrak{m}(S) \mathbb{1}\right)
$$

the negative powers $\left(S_{F}-\mathfrak{m}(S) \mathbb{1}\right)^{-\delta}, \delta>0$, are naturally defined as self-adjoint operators on the Hilbert subspace $\overline{\operatorname{ran}\left(S_{F}-\mathfrak{m}(S) \mathbb{1}\right)}$, or also on the whole $\mathcal{H}$ upon extension by zero on $\operatorname{ker}\left(S_{F}-\mathfrak{m}(S) \mathbb{1}\right)$.

In the first statement we characterise the occurrence of non-Friedrichs extensions with the same Friedrichs lower bound.

Theorem 2.4 Let $S$ be a densely defined and symmetric operator on a given Hilbert space $\mathcal{H}$ with lower bound $\mathfrak{m}(S)>0$. Necessary and sufficient condition for $S$ to admit self-adjoint extensions other then the Friedrichs extensions and with the same lower bound $\mathfrak{m}(S)$ is that

$$
\operatorname{ran}\left(S_{F}-\mathfrak{m}(S) \mathbb{1}\right)^{1 / 2} \cap \operatorname{ker} S^{*} \neq\{0\}
$$

In the applications both $\operatorname{ker} S^{*}$ and $\operatorname{ran}\left(S_{F}-\mathfrak{m}(S) \mathbb{1}\right)^{1 / 2}$ are in general spaces that one can qualify rather explicitly. Thus, condition (2.9) is practically manageable and qualifies the operator-theoretic mechanism for non-Friedrichs extensions with the Friedrichs lower bound. In Sect. 3 we shall give examples of that.

Our next result concerns the actual recipe to construct such extensions, when (2.9) is matched, thus in practice how to identify the corresponding extension parameters $T$ in $\mathcal{S}\left(\operatorname{ker} S^{*}\right)$. We shall use the customary notation of square brackets for the domain $\mathcal{D}[q]$ of a quadratic form $q$ on $\mathcal{H}$ and for the evaluation $q[v]$ on elements of its domain; as usual, we shall denote by $q\left[v_{1}, v_{2}\right]$ the evaluation of the corresponding sesquilinear form defined by polarisation.

Theorem 2.5 Same assumptions as in Theorem 2.4, and assume further that condition (2.9) is satisfied. 
(i) The expression

$$
\begin{aligned}
\mathcal{D}[q] & :=\operatorname{ran}\left(S_{F}-\mathfrak{m}(S) \mathbb{1}\right)^{\frac{1}{2}} \cap \operatorname{ker} S^{*} \\
q[v] & :=\mathfrak{m}(S)\|v\|^{2}+\mathfrak{m}(S)^{2}\left\|\left(S_{F}-\mathfrak{m}(S) \mathbb{1}\right)^{-\frac{1}{2}} v\right\|^{2} .
\end{aligned}
$$

defines a symmetric, closed, and strictly positive quadratic form q.

(ii) Let $T_{q}$ be the operator on the Hilbert subspace $\overline{\mathcal{D}[q]}$ uniquely associated with $q$. Then $T_{q} \in \mathcal{S}\left(\operatorname{ker} S^{*}\right)$.

(iii) For any $T \in \mathcal{S}\left(\operatorname{ker} S^{*}\right)$ with $T \geq T_{q}$, the corresponding self-adjoint extension $S_{T}$ of $S$ (Theorem 2.1) has the property

$$
\mathfrak{m}\left(S_{T}\right)=\mathfrak{m}(S) .
$$

(iv) Any self-adjoint extension $S_{T}$ of $S$ satisfying (2.11) corresponds to an extension parameter $T \in \mathcal{S}\left(\operatorname{ker} S^{*}\right)$ with $T \geq T_{q}$.

By definition, in (2.10) the vector $u_{\circ}=\left(S_{F}-\mathfrak{m}(S) \mathbb{1}\right)^{-\frac{1}{2}} v$ is the minimal norm solution $u=u_{\circ}$ to $\left(S_{F}-\mathfrak{m}(S) \mathbb{1}\right)^{\frac{1}{2}} u=v$.

In view of the general classification of Theorem 2.1, the above results admit a natural corollary that it is worth stating as a separate theorem. It is convenient to introduce the meaningful terminology 'top extensions' for all those $S_{T}$ 's with $\mathfrak{m}\left(S_{T}\right)=\mathfrak{m}(S)$ (in particular, $S_{F}$ is a top extension), and 'least-top extension' for the extension $S_{L T}:=$ $S_{T_{q}}$.

Theorem 2.6 Same assumptions as in Theorem 2.4. Each top extension $S^{\text {top }}$ of $S$ satisfies

$$
S_{F} \geq S^{t o p} \geq S_{L T}
$$

in the sense of operator form ordering. Each such extension is of the form $S^{\text {top }}=S_{T}$ for some $T \in \mathcal{S}\left(\operatorname{ker} S^{*}\right)$ with $T \geq T_{q}$, where $T_{q}$ is qualified in Theorem 2.5(ii), and they are all ordered with $T$ in the sense of (2.4).

Proof (Proof of Theorem 2.4, necessity part)

Let $S_{T}$ be a self-adjoint extension of $S$, labelled by some $T \in \mathcal{S}\left(\operatorname{ker} S^{*}\right)$, with the property $\mathfrak{m}\left(S_{T}\right)=\mathfrak{m}\left(S_{F}\right)$ and $S_{T} \neq S_{F}$. Let $\left(\mu_{n}\right)_{n \in \mathbb{N}}$ be an increasing sequence of real numbers such that $\mu_{n}<\mathfrak{m}(S) \forall n$ and $\mu_{n} \rightarrow \mathfrak{m}(S)$ as $n \rightarrow \infty$. Since $S_{T} \geq \mu_{n} \mathbb{1}$, Theorem 2.2 implies

$$
\langle v, T v\rangle \geq \mu_{n}\|v\|^{2}+\mu_{n}^{2}\left\|\left(S_{F}-\mu_{n} \mathbb{1}\right)^{-\frac{1}{2}} v\right\|^{2}
$$

for every $v \in \mathcal{D}(T)$, whence

$$
\limsup _{n \rightarrow \infty}\left\|\left(S_{F}-\mu_{n} \mathbb{1}\right)^{-\frac{1}{2}} v\right\|^{2}<+\infty .
$$


In fact, for each $v$ the sequence of square norms $\left\|\left(S_{F}-\mu_{n} \mathbb{1}\right)^{-\frac{1}{2}} v\right\|^{2}$ is monotone increasing. For, if $\mathfrak{m}(S)>\mu^{\prime}>\mu$, then

$$
\begin{aligned}
\left\|\left(S_{F}-\mu^{\prime} \mathbb{1}\right)^{-\frac{1}{2}} v\right\|^{2} & -\left\|\left(S_{F}-\mu \mathbb{1}\right)^{-\frac{1}{2}} v\right\|^{2} \\
& =\int_{[\mathfrak{m}(S),+\infty)}\left(\frac{1}{\lambda-\mu^{\prime}}-\frac{1}{\lambda-\mu}\right) \mathrm{d} v_{v}(\lambda) \geq 0,
\end{aligned}
$$

where $v_{v}$ is the scalar spectral measure of the self-adjoint operator $S_{F}$ relative to the vector $v$. Therefore,

$$
\exists \lim _{n \rightarrow \infty}\left\|\left(S_{F}-\mu_{n} \mathbb{1}\right)^{-\frac{1}{2}} v\right\|^{2}<+\infty
$$

As the latter conclusion is tantamount as

$$
\exists \lim _{n \rightarrow \infty} \int_{[\mathfrak{m}(S),+\infty)} \frac{1}{\lambda-\mu_{n}} \mathrm{~d} v_{v}(\lambda)<+\infty,
$$

then by monotone convergence the function $\lambda \mapsto(\lambda-\mathfrak{m}(S))^{-1}$ is $v_{v}$-summable. Thus, $\left\|\left(S_{F}-\mathfrak{m}(S) \mathbb{1}\right)^{-\frac{1}{2}} v\right\|^{2}<+\infty$, whence $v \in \operatorname{ran}\left(S_{F}-\mathfrak{m}(S) \mathbb{1}\right)^{\frac{1}{2}}$.

On the other hand, since by assumption $S_{T}$ is a self-adjoint extension of $S$ distinct from $S_{F}$, then by definition of extension parameter $T$ one has that $\mathcal{D}(T)$ is a non-trivial subspace of ker $S^{*}$. Summarising,

$$
\mathcal{D}(T) \subset \operatorname{ran}\left(S_{F}-\mathfrak{m}(S) \mathbb{1}\right)^{\frac{1}{2}} \cap \operatorname{ker} S^{*}
$$

and therefore $\operatorname{ran}\left(S_{F}-\mathfrak{m}(S) \mathbb{1}\right)^{\frac{1}{2}} \cap \operatorname{ker} S^{*}$ is non-trivial.

Corollary 2.7 As a consequence of the necessity statement of Theorem 2.4, for each self-adjoint extension $S_{T}$ of $S$ with $\mathfrak{m}\left(S_{T}\right)=\mathfrak{m}(S)$ one has

$$
\begin{aligned}
\mathcal{D}(T) & \subset \operatorname{ran}\left(S_{F}-\mathfrak{m}(S) \mathbb{1}\right)^{\frac{1}{2}} \cap \operatorname{ker} S^{*} \quad \text { and } \\
\langle v, T v\rangle & \geq \mathfrak{m}(S)\|v\|^{2}+\mathfrak{m}(S)^{2}\left\|\left(S_{F}-\mathfrak{m}(S) \mathbb{1}\right)^{-\frac{1}{2}} v\right\|^{2} \quad \forall v \in \mathcal{D}(T) .
\end{aligned}
$$

Proof The inclusion for $\mathcal{D}(T)$ was already proved. Next, as a follow-up of the reasoning of the previous proof, let us observe that for each $v \in \mathcal{D}(T)$ one has

$$
\lim _{n \rightarrow \infty}\left\|\left(S_{F}-\mu_{n} \mathbb{1}\right)^{-\frac{1}{2}} v\right\|^{2}=\left\|\left(S_{F}-\mathfrak{m}(S) \mathbb{1}\right)^{-\frac{1}{2}} v\right\|^{2} .
$$

Indeed,

$$
\begin{aligned}
& \left\|\left(S_{F}-\mathfrak{m}(S) \mathbb{1}\right)^{-\frac{1}{2}} v\right\|^{2}-\left\|\left(S_{F}-\mu_{n} \mathbb{1}\right)^{-\frac{1}{2}} v\right\|^{2} \\
& \quad=\int_{[\mathfrak{m}(S),+\infty)}\left(\frac{1}{\lambda-\mathfrak{m}(S)}-\frac{1}{\lambda-\mu_{n}}\right) \mathrm{d} v_{v}(\lambda) \stackrel{n \rightarrow \infty}{\longrightarrow} 0
\end{aligned}
$$


by dominated convergence. Therefore, one can take the limit $n \rightarrow \infty$ in the inequality

$$
\langle v, T v\rangle \geq \mu_{n}\|v\|^{2}+\mu_{n}^{2}\left\|\left(S_{F}-\mu_{n} \mathbb{1}\right)^{-\frac{1}{2}} v\right\|^{2}
$$

thus obtaining the second line of (2.13).

Proof (Proof of Theorem 2.5 and of Theorem 2.4, sufficiency part)

(i) The fact that (2.10) defines a symmetric quadratic form with strictly positive lower bound is obvious. As for $q$ being closed, let us show that if $\left(v_{n}\right)_{n \in \mathbb{N}}$ is a sequence in $\mathcal{D}[q]$ with $v_{n} \rightarrow v$ and $q\left[v_{n}-v_{m}\right] \rightarrow 0$ as $n, m \rightarrow \infty$, then $v \in \mathcal{D}[q]$ and $q\left[v_{n}-v\right] \rightarrow 0$. This is indeed equivalent to saying that $q$ is closed (see, e.g., [11, Prop. 10.1]). Now, the above assumption on $\left(v_{n}\right)_{n \in \mathbb{N}}$ implies

$$
\begin{aligned}
v_{n} & \rightarrow v \\
\left(S_{F}-\mathfrak{m}(S) \mathbb{1}\right)^{-\frac{1}{2}} v_{n} & \rightarrow u
\end{aligned}
$$

for some $v \in \mathcal{H}$. As $\left(S_{F}-\mathfrak{m}(S) \mathbb{1}\right)^{-\frac{1}{2}}$ is self-adjoint and hence closed, this implies

$$
\begin{aligned}
& v \in \mathcal{D}\left(\left(S_{F}-\mathfrak{m}(S) \mathbb{1}\right)^{-\frac{1}{2}}\right)=\operatorname{ran}\left(\left(S_{F}-\mathfrak{m}(S) \mathbb{1}\right)^{\frac{1}{2}}\right) \\
& u=\left(S_{F}-\mathfrak{m}(S) \mathbb{1}\right)^{-\frac{1}{2}} v .
\end{aligned}
$$

A first conclusion, since $\operatorname{ker} S^{*}$ is closed in $\mathcal{H}$ and hence $v \in \operatorname{ker} S^{*}$ as well, is that $v \in \operatorname{ran}\left(\left(S_{F}-\mathfrak{m}(S) \mathbb{1}\right)^{\frac{1}{2}}\right) \cap \operatorname{ker} S^{*}=\mathcal{D}[q]$. As further conclusion, since $v_{n} \rightarrow v$ and $\left(S_{F}-\mathfrak{m}(S) \mathbb{1}\right)^{-\frac{1}{2}} v_{n} \rightarrow\left(S_{F}-\mathfrak{m}(S) \mathbb{1}\right)^{-\frac{1}{2}} v$ in $\mathcal{H}$, one has $q\left[v_{n}-v\right] \rightarrow 0$. Part (i) of Theorem 2.5 is thus proved.

(ii) As $q$ is densely defined in the Hilbert subspace $\overline{\mathcal{D}[q]}$, and it is symmetric, closed, and semi-bounded from below, then $q$ uniquely identifies a self-adjoint operator $T_{q}$ on $\overline{\mathcal{D}[q]}$ defined by

$$
\begin{aligned}
\mathcal{D}\left(T_{q}\right) & :=\left\{v \in \mathcal{D}[q] \mid \exists z_{v} \in \mathcal{H} \text { with }\left\langle u, z_{v}\right\rangle=q[u, v] \forall u \in \mathcal{D}[q]\right\} \\
T_{q} v & :=z_{v}
\end{aligned}
$$

(see, e.g., [11, Theorem 10.7]). Since $\mathcal{D}[q] \subset \operatorname{ker} S^{*}$ and $\operatorname{ker} S^{*}$ is closed in $\mathcal{H}$, then $\overline{\mathcal{D}[q]} \subset \operatorname{ker} S^{*}$, thus proving that $T_{q} \in \mathcal{S}\left(\operatorname{ker} S^{*}\right)$. This establishes part (ii) of Theorem 2.5 .

(iii) Let $T \in \mathcal{S}\left(\operatorname{ker} S^{*}\right)$ with $T \geq T_{q}$. This means that $\mathcal{D}(T) \subset \mathcal{D}\left(T_{q}\right)$ and

$$
\langle v, T v\rangle \geq\left\langle v, T_{q} v\right\rangle=q[v]=\mathfrak{m}(S)\|v\|^{2}+\mathfrak{m}(S)^{2}\left\|\left(S_{F}-\mathfrak{m}(S) \mathbb{1}\right)^{-\frac{1}{2}} v\right\|^{2}
$$

for every $v \in \mathcal{D}(T)$. Consider now an arbitrary $\mu<\mathfrak{m}(S)$. With the very same argument used in the proof of the necessity part of Theorem 2.5 one sees that

$$
\left\|\left(S_{F}-\mathfrak{m}(S) \mathbb{1}\right)^{-\frac{1}{2}} v\right\|^{2}>\left\|\left(S_{F}-\mu \mathbb{1}\right)^{-\frac{1}{2}} v\right\|^{2},
$$


whence

$$
\langle v, T v\rangle>\mu\|v\|^{2}+\mu^{2}\left\|\left(S_{F}-\mu \mathbb{1}\right)^{-\frac{1}{2}} v\right\|^{2}
$$

for all $v \in \mathcal{D}(T)$. Owing to Theorem 2.2, the self-adjoint extension $S_{T}$ of $S$ parametrised by the considered $T$ is such that $S_{T} \geq \mu \mathbb{1}$. By the arbitrariness of $\mu$, one concludes that $S_{T} \geq \mathfrak{m}(S) \mathbb{1}$, whence $\mathfrak{m}\left(S_{T}\right)=\mathfrak{m}(S)$. Unless " $T=\infty$ " (in the sense $\mathcal{D}[T]=\{0\}$ ), all other choices for $T$ identifies non-Friedrichs extensions. This completes the proof of part (iii) of Theorem 2.5. At the same time, this proves that assumption (2.9) in Theorem 2.4 allows one to construct non-Friedrichs extensions with the same Friedrichs lower bound. Thus also the sufficiency statement of Theorem 2.4 is established.

(iv) Last, let $S_{T}$ be a self-adjoint extension of $S$ with $\mathfrak{m}\left(S_{T}\right)=\mathfrak{m}(S)$. The necessity statement of Theorem 2.4 implies that the intersection $\operatorname{ran}\left(S_{F}-\mathfrak{m}(S) \mathbb{1}\right)^{\frac{1}{2}} \cap \operatorname{ker} S^{*}$ is non-trivial, so one can define the form $q$ and the operator $T_{q} \in \mathcal{S}\left(\operatorname{ker} S^{*}\right)$ as in parts (i) and (ii) of Theorem 2.5. Owing to Corollary 2.7,

$$
\begin{aligned}
\mathcal{D}[T] & \subset \mathcal{D}(q) \\
T[v] & =q[v] \quad \forall v \in \mathcal{D}[T] .
\end{aligned}
$$

This means precisely that $T \geq T_{q}$.

\section{Applications}

Let us discuss now a few instructive examples of application of Theorems 2.4 and 2.5. We present examples with deficiency index equal to two (Sect. 3.1) and one (Sects. 3.2, $3.3,3.4$ ), and we cover also the edge case in which the sub-family of top extensions consists of the Friedrichs extension only (Sect. 3.4).

\subsection{Schrödinger Quantum Particle on an Interval}

Let us revisit in more systematic terms the example presented in Sect. 1. The operator $S$ has deficiency index equal to 2 , and explicitly

$$
\operatorname{ker} S^{*}=\operatorname{span}\{\mathbf{1}, x\} \text {. }
$$

The operator $S_{F}-\pi^{2} \mathbb{1}$ fails to be invertible on the whole $\mathcal{H}=L^{2}(0,1)$ because it has a non-trivial kernel:

$$
\begin{aligned}
& \operatorname{ker}\left(S_{F}-\pi^{2} \mathbb{1}\right)=\operatorname{span}\{\sin \pi x\} \\
& \operatorname{ran}\left(S_{F}-\pi^{2} \mathbb{1}\right)=\operatorname{span}\{\sin n \pi x \mid n \in \mathbb{N}, n \geq 2\} .
\end{aligned}
$$


As $\left(S_{F}-\pi^{2} \mathbb{1}\right)$ is diagonalised as above over an orthonormal basis of eigenfunctions, its powers $\left(S_{F}-\pi^{2} \mathbb{1}\right)^{\delta}$ and $\left(S_{F}-\pi^{2} \mathbb{1}\right)^{-\delta}$, with $\delta>0$, are qualified by their action on the same basis of eigenfunctions, with eigenvalues given by the corresponding powers of the eigenvalues of $\left(S_{F}-\pi^{2} \mathbb{1}\right)$; the negative powers are clearly only defined on the Hilbert subspace $\overline{\operatorname{ran}\left(S_{F}-\pi^{2} \mathbb{1}\right)}=\{\sin \pi x\}^{\perp}$. Therefore,

$$
\operatorname{ran}\left(S_{F}-\pi^{2} \mathbb{1}\right)^{\frac{1}{2}}=\operatorname{ran}\left(S_{F}-\pi^{2} \mathbb{1}\right)=\operatorname{span}\{\sin n \pi x \mid n \in \mathbb{N}, n \geq 2\} .
$$

Lemma 3.1 One has

$$
V:=\operatorname{ran}\left(S_{F}-\pi^{2} \mathbb{1}\right)^{\frac{1}{2}} \cap \operatorname{ker} S^{*}=\operatorname{span}\{\mathbf{1}-2 x\}
$$

and

$$
\left(S_{F}-\pi^{2} \mathbb{1}\right)^{-1}(\mathbf{1}-2 x)=\pi^{-2}(\cos \pi x-\mathbf{1}+2 x) .
$$

Proof In order for a generic element $a \mathbf{1}+b x \in \operatorname{ker} S^{*}$, with $a, b \in \mathbb{C}$, to belong to $\operatorname{ran}\left(S_{F}-\pi^{2} \mathbb{1}\right)^{\frac{1}{2}}$, owing to (3.2)-(3.3) it must be

$$
0=\int_{0}^{1}(a+b x) \sin \pi x \mathrm{~d} x=\pi^{-1}(2 a+b),
$$

whence $b=-2 a$. Thus $g \in V$ implies $g=a(\mathbf{1}-2 x)$ for some $a \in \mathbb{C}$. Next, one has to check that $1-2 x \in \operatorname{ran}\left(S_{F}-\pi^{2} \mathbb{1}\right)^{\frac{1}{2}}$. This is the same as $\mathbf{1}-2 x \in \operatorname{ran}\left(S_{F}-\pi^{2} \mathbb{1}\right)$, that is,

$$
\mathbf{1}-2 x=\left(S_{F}-\pi^{2} \mathbb{1}\right) u \quad \text { for some } u \in \mathcal{D}\left(S_{F}\right) .
$$

This is equivalent to saying that $u$ is the minimal norm solution to the boundary value problem

$$
\left\{\begin{array}{l}
-u^{\prime \prime}-\pi^{2} u=1-2 x \\
u(0)=0=u(1)
\end{array}\right.
$$

By standard ODE methods one finds that the general solution is

$$
u_{\mathrm{gen}}(x)=\pi^{-2}(\cos \pi x-1+2 x)+B \sin \pi x, \quad B \in \mathbb{C},
$$

thus the minimal norm solution is the one with $B=0$. This proves that the function $u_{\circ}:=\pi^{-2}(\cos \pi x-\mathbf{1}+2 x) \in \mathcal{D}\left(S_{F}\right)$ satisfies $\left(S_{F}-\pi^{2} \mathbb{1}\right) u_{\circ}=\mathbf{1}-2 x$, thus completing the proof of (3.4) and (3.5).

As the intersection space (3.4) is non-trivial, Theorem 2.4 ensures that $S$ admits non-Friedrichs self-adjoint extensions with the same Friedrichs lower bound. This is 
consistent with what discussed in the introduction: $\mathfrak{m}\left(S_{F}\right)=\mathfrak{m}\left(S_{A}\right)$, Friedrichs and anti-periodic extension have the same lower bound.

It is instructive to apply the constructive recipe of Theorem 2.5 so as to identify all such extensions. With the notation therein,

$$
\mathcal{D}[q]=\mathcal{D}\left(T_{q}\right)=V=\operatorname{span}\{\mathbf{1}-2 x\}
$$

thus $T_{q}$ is an operator of multiplication by some real number $t_{q}$,

$$
T_{q}(\mathbf{1}-2 x)=t_{q}(\mathbf{1}-2 x) .
$$

Since

$$
\begin{aligned}
\left\langle(\mathbf{1}-2 x), T_{q}(\mathbf{1}-2 x)\right\rangle & =\pi^{2}\|\mathbf{1}-2 x\|_{2}^{2}+\pi^{4}\left\|\left(S_{F}-\pi^{2} \mathbb{1}\right)^{-\frac{1}{2}}(\mathbf{1}-2 x)\right\|_{2}^{2} \\
& =\pi^{2}\|\mathbf{1}-2 x\|_{2}^{2}+\pi^{4}\left\langle(\mathbf{1}-2 x),\left(S_{F}-\pi^{2} \mathbb{1}\right)^{-1}(\mathbf{1}-2 x)\right\rangle \\
& \left.=\pi^{2}\|\mathbf{1}-2 x\|_{2}^{2}+\pi^{2}\langle(\mathbf{1}-2 x), \cos x-(\mathbf{1}-2 x))\right\rangle \\
& =\pi^{2}\langle(\mathbf{1}-2 x), \cos x\rangle \\
& =4=12\|\mathbf{1}-2 x\|_{2}^{2}
\end{aligned}
$$

(having used (3.5) in the third step and $\|\mathbf{1}-2 x\|_{2}^{2}=\frac{1}{3}$ in the last step), then necessarily $t_{q}=12$.

Theorem 2.5, in parts (iii) and (iv), then states that the self-adjoint extensions $S_{T}$ of $S$ with $\mathfrak{m}\left(S_{T}\right)=\mathfrak{m}\left(S_{F}\right)$ are those labelled by self-adjoint operators $T$ with $T \geq T_{q}$. Such $T$ 's, apart from the one parametrising the Friedrichs extension, are therefore such that

$$
\mathcal{D}(T)=V=\operatorname{span}\{\mathbf{1}-2 x\}
$$

$T$ is the multiplication by somet $\geq 12$.

Keeping into account, as is immediate to check, that

$$
W:=V^{\perp} \cap \operatorname{ker} S^{*}=\operatorname{span}\{\mathbf{1}\}
$$

the extension $S_{T}$ for each $T$ satisfying (3.8) has domain given by formula (2.1) of Theorem 2.1, that is,

$$
\begin{aligned}
& \mathcal{D}\left(S_{T}\right)=\left\{\begin{array}{l|l}
f+S_{F}^{-1}(T v+w)+v \mid \begin{array}{c}
f \in \mathcal{D}(\bar{S}), \\
v \in V, w \in W
\end{array}
\end{array}\right\} \\
& =\left\{\begin{array}{l|l}
f+S_{F}^{-1}(t \alpha(\mathbf{1}-2 x)+\beta \mathbf{1})+\alpha(\mathbf{1}-2 x) & \begin{array}{c}
f \in H_{0}^{2}(0,1) \\
\alpha, \beta \in \mathbb{C}
\end{array}
\end{array}\right\} .
\end{aligned}
$$

The action of the everywhere defined and bounded operator $S_{F}^{-1}$ on the subspace $\operatorname{ker} S^{*}=\operatorname{span}\{\mathbf{1}, x\}$ is easily computed by solving a boundary value problem completely analogous to the one considered in the proof of Lemma 3.1. The result (as 
found, e.g., in [5, Eq. (91)]) is

$$
S_{F}^{-1}(a \mathbf{1}+b x)=\left(\frac{a}{2}+\frac{b}{6}\right) x-\frac{a}{2} x^{2}-\frac{b}{6} x^{3} .
$$

Thus,

$$
\mathcal{D}\left(S_{T}\right)=\left\{\begin{array}{ll}
f+\alpha \mathbf{1}+\left(\frac{t \alpha+3 \beta}{6}-2 \alpha\right) x-\frac{t \alpha+\beta}{2} x^{2}+\frac{t \alpha}{3} x^{3} \mid \begin{array}{l}
f \in H_{0}^{2}(0,1) \\
\alpha, \beta \in \mathbb{C}
\end{array}
\end{array}\right\}
$$

Formula (3.12), for each fixed $t \geq 12$, identifies those self-adjoint extensions of $S$ different from the Friedrichs extension, but with the same lower bound. In order to identify the boundary condition of self-adjointness satisfied by a generic element $g \in \mathcal{D}\left(S_{T}\right)$ for each extension of type (3.12), we compute the boundary values

$$
\begin{array}{ll}
g(0)=\alpha & g^{\prime}(0)=\frac{t \alpha}{6}+\frac{\beta}{2}-2 \alpha \\
g(1)=-\alpha & g^{\prime}(1)=\frac{t \alpha}{6}-\frac{\beta}{2}-2 \alpha
\end{array}
$$

and re-write

$$
\begin{aligned}
g(0)+g(1) & =0 \\
g^{\prime}(0)+g^{\prime}(1) & =\frac{1}{3}(t-12) g(0) .
\end{aligned}
$$

It was indeed convenient to cast (3.13) in the form (3.14) because the latter can be more easily matched with the general conditions of self-adjointness of the extensions of $S$, as we shall now do.

We refer to the following very standard result, obtained for example by exploiting Theorem 2.1 for all possible extension parameters (see, e.g., [11, Example 14.10]), or equivalently by means of the alternative extension scheme a la von Neumann applied to $S$ (see, e.g., [6, Sect. 6.2.3.1]).

Proposition 3.2 The family of self-adjoint extensions on $L^{2}(0,1)$ of the operator $S$ defined in (1.1) consists of restrictions of $S^{*}$, and hence of operators of the form $-\frac{d^{2}}{d x^{2}}$, to domains of $H^{2}(0,1)$-functions $g$ satisfying boundary conditions of one of the following four classes:

$$
\begin{array}{r}
g^{\prime}(0)=b_{1} g(0)+c g(1), \quad g^{\prime}(1)=-\bar{c} g(0)-b_{2} g(1), \\
g^{\prime}(0)=b_{1} g(0)+\bar{c} g^{\prime}(1), \quad g(1)=c g(0), \\
g^{\prime}(1)=-b_{1} g(1), \quad g(0)=0, \\
g(0)=0=g(1),
\end{array}
$$

where $c \in \mathbb{C}$ and $b_{1}, b_{2} \in \mathbb{R}$ and qualify each extension. 
Remark 3.3 With reference to the general formula (2.1), extensions of type (3.15) correspond to the case in which $\operatorname{dim} \mathcal{D}(T)=2$, extensions of type (3.16) or (3.17) correspond to $\operatorname{dim} \mathcal{D}(T)=1$, and the extension of type (3.18) is the Friedrichs extension, $\operatorname{dim} \mathcal{D}(T)=0$.

By direct comparison between (3.14) and (3.15)-(3.18) we see that (3.14) can only be of type (3.16) with

$$
b_{1}=\frac{1}{3}(t-12) \quad \text { and } \quad c=-1 .
$$

We have thus proved the following.

Proposition 3.4 The non-Friedrichs self-adjoint extensions on $L^{2}(0,1)$ of the operator $S$ defined in (1.1) which preserve the Friedrichs lower bound $\mathfrak{m}(S)=\pi^{2}$ are all those operators acting as $-\frac{d^{2}}{d x^{2}}$ on $H^{2}(0,1)$-functions $g$ with boundary condition

$$
\begin{aligned}
g(0)+g(1) & =0 \\
g^{\prime}(0)+g^{\prime}(1) & =b g(0)
\end{aligned}
$$

for fixed $b \geq 0$. Each $b$ qualifies one of such extensions, with a one-to-one correspondence. Such extensions are ordered with increasing $b$. The choice $b=0$ corresponds to anti-periodic boundary conditions.

The application of Theorems 2.4 and 2.5 thus allowed for a fast identification of all non-Friedrichs extensions with Friedrichs lower bound of the minimally defined Laplacian on $[0,1]$, which would have otherwise required a tedious computation, by means of (3.15)-(3.18), of all the discrete spectra of the various extensions, in order to select those with bottom equal to $\pi^{2}$.

For completeness, here is how the direct check would have proceeded. Let us limit the analysis to the eigenvalue problem for a generic self-adjoint extension of type (3.16) with the choice (3.19), namely

$$
\left\{\begin{array}{l}
-g^{\prime \prime}=\lambda g \\
g(0)+g(1)=0 \\
g^{\prime}(0)+g^{\prime}(1)=\frac{1}{3}(t-12) g(0) \\
\left(\lambda \in \mathbb{R}, g \in H^{2}(0,1)\right)
\end{array}\right.
$$

for fixed $t \in \mathbb{R}$. $g$ must be of the form $g(x)=A \cos \sqrt{\lambda} x+B \sin \sqrt{\lambda} x, A, B \in \mathbb{C}$, and for sure the pairs $(g, \lambda)$ with

$$
g(x)=\sin ((2 n+1) \pi x), \quad \lambda=(2 n+1)^{2} \pi^{2}, \quad n \in \mathbb{N}_{0}
$$

solve (3.21), showing that all such extensions have the eigenvalues $(2 n+1)^{2} \pi^{2}$, $n \in \mathbb{N}_{0}$, in common. The remaining (i.e., non-sin-only) solutions to (3.21) are obtained 

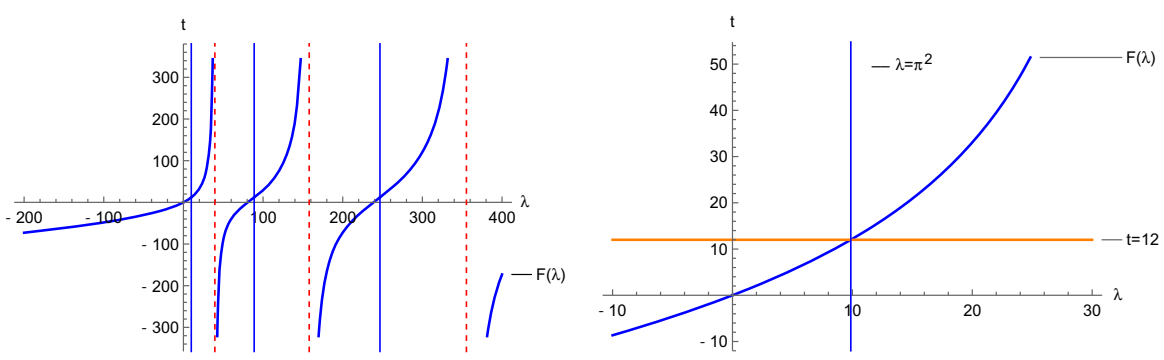

Fig. 1 Left: plot of $F(\lambda)$ defined in (3.23) (blue curves) as compared to the eigenvalues of type (3.22) (vertical blue lines) and the Friedrichs eigenvalues (dashed red lines). Right: magnification of the first positive interval of definition of $F(\lambda)$. Eigenvalues determined by (3.22) and (3.23) correspond to the intersections of the blue curves with the horizontal lines at level $t$ (Color figure online)

imposing $B \neq 0$, and it is then simple to conclude that the admissible $\lambda$ 's are the ( $t$ dependent) roots of

$$
F(\lambda)=t, \quad \text { where } \quad F(\lambda):=12-6 \sqrt{\lambda} \frac{1+\cos \sqrt{\lambda}}{\sin \sqrt{\lambda}}
$$

(and understanding the above trigonometric functions as hyperbolic functions when $\lambda<0)$. As $F(\lambda)$ increases with $\lambda$ in all intervals in which it is defined, and $F\left(\pi^{2}\right)=12$, one deduces that only for $t \geq 12$ the admissible $\lambda$ 's selected by (3.23) satisfy $\lambda \geq \pi^{2}$ (see Fig. 1). The spectrum thus determined from (3.22) and (3.23) indeed confirms, by direct inspection, what found in Prop. 3.4 by means of our Theorem 2.5.

\subsection{Schrödinger Quantum Particle in $\mathbb{R}^{3}$ with Point Interaction}

This is an example with deficiency index equal to 1 . With respect to the Hilbert space $\mathcal{H}=L^{2}\left(\mathbb{R}^{3}\right)$ we consider the operator

$$
\mathcal{D}(\tilde{S})=C_{0}^{\infty}\left(\mathbb{R}^{3} \backslash\{0\}\right), \quad \tilde{S}=-\Delta .
$$

$\widetilde{S}$ is densely defined and symmetric, with $\mathfrak{m}(\widetilde{S})=0$.

The self-adjoint extensions of $\widetilde{S}$ are Hamiltonians for a quantum particle in three dimensions subject to a point interaction supported at $x=0$, and they are very well studied and understood.

Theorem 3.5 [See, e.g., [1, Chapt. I.1].]

(i) $\widetilde{S}$ has unit deficiency index. The Friedrichs extension of $\widetilde{S}$ is the self-adjoint (negative) Laplacian on $L^{2}(0,1)$ with domain $H^{2}(0,1)$. All other self-adjoint extensions of $\widetilde{S}$ form the family $\left\{-\Delta_{\alpha} \mid \alpha \in \mathbb{R}\right\}$, where

$$
\begin{aligned}
\mathcal{D}\left(-\Delta_{\alpha}\right) & =\left\{g=\phi+\frac{\phi(0)}{\alpha+\frac{1}{4 \pi}} G_{1} \mid \phi \in H^{2}\left(\mathbb{R}^{3}\right)\right\} \\
\left(-\Delta_{\alpha}+\mathbb{1}\right) g & =(-\Delta+\mathbb{1}) \phi
\end{aligned}
$$


and

$$
G_{1}:=(2 \pi)^{\frac{3}{2}} \frac{e^{-|x|}}{4 \pi|x|} .
$$

(ii) For each $\alpha \in \mathbb{R}$,

$$
\sigma_{e s s}\left(-\Delta_{\alpha}\right)=\sigma_{a c}\left(-\Delta_{\alpha}\right)=[0,+\infty), \quad \sigma_{s c}\left(-\Delta_{\alpha}\right)=\emptyset,
$$

and

$$
\sigma_{p}\left(-\Delta_{\alpha}\right)=\left\{\begin{array}{cl}
\emptyset & \text { if } \alpha \in[0,+\infty] \\
\left\{-(4 \pi \alpha)^{2}\right\} & \text { if } \alpha \in(-\infty, 0)
\end{array}\right.
$$

The negative eigenvalue $-(4 \pi \alpha)^{2}$, when it exists, is simple and the corresponding eigenfunction is $|x|^{-1} e^{4 \pi \alpha|x|}$.

We see from Theorem 3.5 that $\widetilde{S}$ admits a collection of non-Friedrichs extensions with Friedrichs lower bound, and precisely

$$
\mathfrak{m}\left(-\Delta_{\alpha}\right)=0=\mathfrak{m}(\widetilde{S}) \quad \forall \alpha \geq 0
$$

In order to recover such a conclusion from the abstract setting of Sect. 2, let us consider

$$
S:=\widetilde{S}+\mathbb{1}
$$

Clearly, $\mathfrak{m}(S)=1$. The self-adjoint extensions of $\widetilde{S}$ and of $S$ then only differ by a trivial shift. As we intend to analyse the extensions of $S$ within the extension scheme of Theorem 2.1, rather than using von Neumann's extension theorem as in [1], let us follow closely the discussion made in [10, Sect. 3], were indeed the Kreĭn-VišikBirman scheme was employed.

We shell denote, respectively, by $\widehat{ }$ and - the Fourier and inverse Fourier transform $L^{2}\left(\mathbb{R}^{3}, \mathrm{~d} x\right) \rightarrow L^{2}\left(\mathbb{R}^{3}, \mathrm{~d} p\right)$ with the convention

$$
\widehat{f}(p)=\frac{1}{(2 \pi)^{\frac{3}{2}}} \int_{\mathbb{R}^{3}} e^{-\mathrm{i} p \cdot x} f(x) \mathrm{d} x .
$$

In particular,

$$
G_{1}=(2 \pi)^{\frac{3}{2}} \frac{e^{-|x|}}{4 \pi|x|}=\left(\frac{1}{p^{2}+1}\right)^{2}
$$

It is possible to prove the following. 
Theorem 3.6 [10, Sect. 3]

(i) S has deficiency space

$$
\operatorname{ker} S^{*}=\operatorname{span}\left\{G_{1}\right\}
$$

(ii) The Friedrichs extension of $S$ is the operator

$$
\begin{aligned}
\mathcal{D}\left(S_{F}\right) & =\left\{g \in L^{2}\left(\mathbb{R}^{3}\right) \mid \begin{array}{c}
\widehat{g}=\widehat{f}+\left(p^{2}+1\right)^{-1} \eta \\
f \in \mathcal{D}(\bar{S}), \eta \in \mathbb{C}
\end{array}\right\} \\
\widehat{S_{F} g} & =\left(p^{2}+1\right) \widehat{g} .
\end{aligned}
$$

(iii) All other self-adjoint extensions of $S$ are of the form $S_{t}$ for some $t \in \mathbb{R}$, where

$$
\begin{aligned}
\mathcal{D}\left(S_{t}\right) & =\left\{g \in L^{2}\left(\mathbb{R}^{3}\right) \mid \begin{array}{c}
\hat{g}=\widehat{f}+\left(p^{2}+1\right)^{-2} t \xi+\left(p^{2}+1\right)^{-1} \xi \\
f \in \mathcal{D}(\bar{S}), \xi \in \mathbb{C}
\end{array}\right\} \\
\widehat{S_{t} g} & =\left(p^{2}+1\right)\left(\widehat{f}+\left(p^{2}+1\right)^{-2} t \xi\right) .
\end{aligned}
$$

This is precisely formula (2.1) of Theorem 2.1 specialised to the case where ker $S^{*}$ is one-dimensional and $T$ is therefore the operator of multiplication by the real number $t$.

(iv) One has

$$
S_{t}=-\Delta_{\alpha}+\mathbb{1} \quad \text { for } \quad \alpha=\frac{t-2}{8 \pi}
$$

Clearly, $S_{F}-\mathfrak{m}(S) \mathbb{1}=S_{F}-\mathbb{1}=\widetilde{S}_{F}$, the self-adjoint (negative) Laplacian on $L^{2}\left(\mathbb{R}^{3}\right)$. Therefore, unlike the example discussed in Sect. 3.1,

$$
\operatorname{ker}\left(S_{F}-\mathbb{1}\right)=\{0\} .
$$

$S_{F}-\mathbb{1}$ is then invertible on its range and so are the powers $\left(S_{F}-\mathbb{1}\right)^{\delta}, \delta>0$. On such a space, $\left(S_{F}-\mathbb{1}\right)^{-\delta}$ acts, in Fourier transform, as the multiplication by $|p|^{-2 \delta}$.

The analogue of Lemma 3.1 is now the following.

Lemma 3.7 One has

$$
V:=\operatorname{ran}\left(S_{F}-\mathbb{1}\right)^{\frac{1}{2}} \cap \operatorname{ker} S^{*}=\operatorname{span}\left\{G_{1}\right\}
$$

and

$$
\left(S_{F}-\mathbb{1}\right)^{-\frac{1}{2}} G_{1}=\left(\frac{1}{|p|\left(p^{2}+1\right)}\right)
$$


Proof The fact that $G_{1} \in \operatorname{ker} S^{*}$ is stated in Theorem 3.6(i). As

$$
\frac{1}{|p|\left(p^{2}+1\right)} \in L^{2}\left(\mathbb{R}^{3}, \mathrm{~d} p\right)
$$

and

$$
\left(S_{F}-\mathbb{1}\right)^{\frac{1}{2}}\left(\frac{1}{|p|\left(p^{2}+1\right)}\right)^{\vee}=\left(\frac{1}{\left(p^{2}+1\right)}\right)^{\vee}=G_{1},
$$

hence $G_{1} \in \operatorname{ran}\left(S_{F}-\mathbb{1}\right)^{\frac{1}{2}} . V$ can be at most one-dimensional, thus (3.37) is proved, and so is (3.38) as well.

Owing to Lemma 3.7, Theorem 2.4 is applicable: $S$ admits non-Friedrichs extensions with Friedrichs lower bound, and so does therefore $\widetilde{S}$, consistently with what previously observed in (3.29).

Furthermore, with the notation of Theorem 2.5,

$$
\mathcal{D}[q]=\mathcal{D}\left(T_{q}\right)=V=\operatorname{span}\left\{G_{1}\right\}
$$

thus $T_{q}$ is an operator of multiplication by some real number $t_{q}$,

$$
T_{q} G_{1}=t_{q} G_{1}
$$

Since

$$
\begin{aligned}
\left\langle G_{1}, T_{q} G_{1}\right\rangle & =\left\|G_{1}\right\|_{2}^{2}+\left\|\left(S_{F}-\mathbb{1}\right)^{-\frac{1}{2}} G_{1}\right\|_{2}^{2} \\
& =\left\|\frac{1}{p^{2}+1}\right\|_{2}^{2}+\left\|\frac{1}{|p|\left(p^{2}+1\right)}\right\|_{2}^{2} \\
& =\pi^{2}+\pi^{2} \\
& =2\left\|G_{1}\right\|_{2}^{2}
\end{aligned}
$$

(having used (3.38) in the second identity), then necessarily $t_{q}=2$.

Theorem 2.5, in parts (iii) and (iv), then states that the self-adjoint extensions $S_{T}$ of $S$ with $\mathfrak{m}\left(S_{T}\right)=\mathfrak{m}\left(S_{F}\right)$ are those labelled by self-adjoint operators $T$ with $T \geq T_{q}$. Such $T$ 's, apart from the one parametrising the Friedrichs extension, are therefore such that

$$
\mathcal{D}(T)=V=\operatorname{span}\left\{G_{1}\right\}
$$

$T$ is the multiplication by some $t \geq 2$.

For what argued in Theorem 3.6(iii), such extensions are precisely the operators $S_{t}$ that one reads out from formula (3.34) with $t \geq 2$. In turn, the correspondence formula (3.35) leads to the conclusion that the self-adjoint extensions of $\widetilde{S}$ with Friedrichs lower bound are precisely those $-\Delta_{\alpha}$ 's with $\alpha \geq 0$. 


\subsection{Radial Problem in Hydrogenoid-Like Hamiltonians}

It is worth mentioning another example with unit deficiency index, even without working out here the steps through which Theorems 2.4 and 2.5 are applied, which are in fact completely analogous to the computations of Sects. 3.1 and 3.2.

For given $v \in \mathbb{R}$, let us now consider

$$
\mathcal{D}\left(S_{v}\right)=C_{0}^{\infty}\left(\mathbb{R}^{+}\right), \quad S_{v}=-\frac{\mathrm{d}^{2}}{\mathrm{~d} x^{2}}+\frac{v}{x},
$$

a densely defined and symmetric operator on the Hilbert space $\mathcal{H}=L^{2}\left(\mathbb{R}^{+}\right)$with lower bound $\mathfrak{m}\left(S_{v}\right)=0$. One typical emergence of $S_{v}$ in mathematical physics is as the minimally defined zero-momentum radial operator in the construction of a quantum hydrogenoid Hamiltonian with an additional point interaction at the center of the Coulomb potential: $S_{v}$ is indeed well known and thoroughly studied, and we refer to [4, Sect. 1.4] and references therein for an updated historical overview.

Hardy's inequality implies that $S_{v}$ is lower semi-bounded, and in particular obviously

$$
\mathfrak{m}\left(S_{v}\right)=0 \quad \forall v \geq 0
$$

(repulsive Coulomb interaction). A standard limit-point limit-circle argument shows that $S_{v}$ has unit deficiency index. Its self-adjoint extensions are studied in the literature by means of various extension schemes, including recently in [4] by means of the general Theorem 2.1 above.

Theorem 3.8 [4, Theorems 2 and 4].

(i) The self-adjoint extensions of $S_{v}$ in $L^{2}\left(\mathbb{R}^{+}\right)$form the family $\left\{S_{v}^{(\alpha)} \mid \alpha \in \mathbb{R} \cup\{\infty\}\right\}$, where $\alpha=\infty$ labels the Friedrichs extension, and

$$
\begin{aligned}
\mathcal{D}\left(S_{v}^{(\alpha)}\right) & =\left\{g \in L^{2}\left(\mathbb{R}^{+}\right) \mid \begin{array}{c}
-g^{\prime \prime}+\frac{v}{r} g \in L^{2}\left(\mathbb{R}^{+}\right) \\
\text {and } g_{1}=4 \pi \alpha g_{0}
\end{array}\right\} \\
S_{v}^{(\alpha)} g & =-g^{\prime \prime}+\frac{v}{r} g,
\end{aligned}
$$

$g_{0}$ and $g_{1}$ being the existing limits

$$
\begin{aligned}
& g_{0}:=\lim _{r \downarrow 0} g(r) \\
& g_{1}:=\lim _{r \downarrow 0} r^{-1}\left(g(r)-g_{0}(1+v r \ln r)\right) .
\end{aligned}
$$

(ii) For $v>0$ one has

$$
\sigma_{e s s}\left(S_{v}^{(\alpha)}\right)=\sigma_{a c}\left(S_{v}^{(\alpha)}\right)=[0,+\infty), \quad \sigma_{s c}\left(S_{v}^{(\alpha)}\right)=\emptyset
$$


and

$$
\sigma_{p}\left(S_{v}^{(\alpha)}\right)=\left\{\begin{array}{cl}
\emptyset & \text { if } \alpha \geq \alpha_{v} \\
\left\{E_{+}^{(v, \alpha)}\right\} & \text { if } \alpha<\alpha_{v}
\end{array}\right.
$$

where

$$
\alpha_{v}:=\frac{v}{4 \pi}(\ln v+2 \gamma-1)
$$

$\left(\gamma \sim 0.577\right.$ being the Euler-Mascheroni constant) and $E_{+}^{(v, \alpha)}$ is the only simple negative root of $\mathfrak{F}_{\nu}(E)=\alpha$ with

$$
\mathfrak{F}_{\nu}(E):=\frac{v}{4 \pi}\left(\psi\left(1+\frac{v}{2 \sqrt{|E|}}\right)+\ln (2 \sqrt{|E|})+2 \gamma-1-\frac{\sqrt{|E|}}{v}\right)
$$

$\left(\psi(z)=\Gamma^{\prime}(z) / \Gamma(z)\right.$ being the digamma function $)$.

When $v>0$ Theorem 3.8 thus shows that

$$
\begin{array}{ll}
\mathfrak{m}\left(S_{v}^{(\alpha)}\right)=E_{+}^{(v, \alpha)}<0=\mathfrak{m}\left(S_{v}\right) & \text { if } \alpha<\alpha_{v} \\
\mathfrak{m}\left(S_{v}^{(\alpha)}\right)=0=\mathfrak{m}\left(S_{v}\right) & \text { if } \alpha \geq \alpha_{v}
\end{array}
$$

yet another example of the presence of a sub-class of non-Friedrichs extensions with Friedrichs lower bound.

Also on this example it is easy to test the applicability of our Theorems 2.4-2.5. As done in Sect. 3.2, since $\mathfrak{m}\left(S_{v}\right)=0$, a positive shift must be performed first. For similar purposes the analysis of the shifted operator

$$
\mathcal{S}_{v}:=S_{v}+\frac{v^{2}}{4 \kappa^{2}} \mathbb{1} \quad(\kappa \in \mathbb{R})
$$

and of its self-adjoint extensions was worked in [4, Sect. 2], which we refer to for the details. The special value of the shift (3.51) was chosen in [4] in order to be able to solve the ODE $\mathcal{S}_{v}^{*} u=0$ by means of special functions, this way characterising explicitly the deficiency space ker $\mathcal{S}_{v}^{*}$. The Friedrichs extension $\mathcal{S}_{v, F}$ of $\mathcal{S}_{v}$ was also characterised in [4, Sect. 2]. This provides all the ingredients to investigate the intersection (2.9) and apply Theorems 2.4 and 2.5 so as to reproduce (3.50).

\subsection{Schrödinger Quantum Particle on an Interval, Continued}

In this last example let us show how our abstract results apply also to the edge case in which no other self-adjoint extension has the same bottom as the Friedrichs'. 
Acting on the Hilbert space $\mathcal{H}=L^{2}(0,1)$, let us consider the densely defined and symmetric operator

$$
S=-\frac{\mathrm{d}^{2}}{\mathrm{~d} x^{2}}, \quad \mathcal{D}(S)=\left\{f \in H^{2}(0,1) \mid f(0)=f^{\prime}(0)=f(1)=0\right\}
$$

Again by Poincaré inequality, $S$ is semi-bounded from below with $\mathfrak{m}(S)=\pi^{2}$.

It is standard to see that $S$ is a symmetric extension of the operator considered in Sect. 3.1, but now with unit deficiency index, with deficiency subspace

$$
\operatorname{ker} S^{*}=\operatorname{span}\{\mathbf{1}-x\}
$$

and with Friedrichs extension $S_{F}$ given exactly by the already considered operator (1.5). Thus, (3.2)-(3.3) above apply, and therefore the spanning function $\mathbf{1}-x$ cannot belong to $\operatorname{ran}\left(S_{F}-\pi^{2} \mathbb{1}\right)^{\frac{1}{2}}=\operatorname{ran}\left(S_{F}-\pi^{2} \mathbb{1}\right)=\{\sin \pi x\}^{\perp}$, for

$$
\int_{0}^{1}(1-x) \sin \pi x d x=\frac{1}{\pi} \neq 0 \text {. }
$$

The conclusion is then

$$
\operatorname{ran}\left(S_{F}-\pi^{2} \mathbb{1}\right)^{\frac{1}{2}} \cap \operatorname{ker} S^{*}=\{0\}
$$

Theorem 2.4 then predicts that $S_{F}$ is the only self-adjoint extension of $S$ with lower bound $\pi^{2}$.

For an independent direct check of the latter conclusion, one can argue that the one-parameter family of self-adjoint extensions of $S$ consists of negative Laplacians on $H^{2}$-functions $g$ subject to the condition (3.16) with $c=0$ and $b_{1} \in \mathbb{R}$, i.e.,

$$
g^{\prime}(0)=b_{1} g(0), \quad g(1)=0 .
$$

The corresponding eigenvalue problem, namely the analogue of (3.21) now with the boundary condition (3.56), is solved by the roots $\lambda \in \mathbb{R}$ of

$$
-\frac{\sqrt{\lambda}}{\tan \sqrt{\lambda}}=b_{1}
$$

for given $b_{1} \in \mathbb{R}$ that qualifies each extension (and understanding (3.57) in terms of the hyperbolic tangent when $\lambda<0$ ). One easily sees (Fig. 2) that (3.57) always admits a solution $\lambda<\pi^{2}=\mathfrak{m}\left(S_{F}\right)$, and only asymptotically for $b_{1} \rightarrow+\infty$ does the smallest root approach $\pi^{2}$, thus proving that each such extension is strictly lower that $S_{F}$. 
Fig. 2 Graphic solution to (3.57)

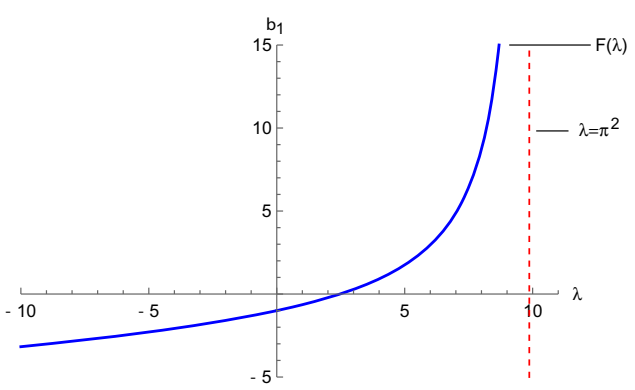

Funding Open access funding provided by Università degli Studi di Milano within the CRUI-CARE Agreement.

Open Access This article is licensed under a Creative Commons Attribution 4.0 International License, which permits use, sharing, adaptation, distribution and reproduction in any medium or format, as long as you give appropriate credit to the original author(s) and the source, provide a link to the Creative Commons licence, and indicate if changes were made. The images or other third party material in this article are included in the article's Creative Commons licence, unless indicated otherwise in a credit line to the material. If material is not included in the article's Creative Commons licence and your intended use is not permitted by statutory regulation or exceeds the permitted use, you will need to obtain permission directly from the copyright holder. To view a copy of this licence, visit http://creativecommons.org/licenses/by/4.0/.

\section{References}

1. Albeverio, S., Gesztesy, F., Høegh-Krohn, R., Holden, H.: Solvable Models in Quantum Mechanics, Texts and Monographs in Physics. Springer, New York (1988)

2. Behrndt, J., Hassi, S., de Snoo, H.: Boundary value problems, Weyl functions, and differential operators. Monographs in Mathematics, vol. 108. Birkhäuser/Springer, Cham (2020)

3. Birman, M v: On the theory of self-adjoint extensions of positive definite operators. Mat. Sb. N.S. 38(80), 431-450 (1956)

4. Gallone, M., Michelangeli, A.: Hydrogenoid spectra withcentral perturbations. Rep. Math. Phys. 84, 215-243 (2019)

5. Gallone, M., Michelangeli, A., Ottolini, A.: Krě̆n-Višik-Birman self-adjoint extension theory revisited, SISSA preprint 25/2017/MATE (2017)

6. Gitman, D.M., Tyutin, I.V., Voronov, B.L.: Self-adjoint extensions in quantum mechanics, vol. 62 of progress in mathematical physics, Birkhäuser/Springer, New York, 2012. General theory and applications to Schrödinger and Dirac equations with singular potentials

7. Grubb, G.: A characterization of the non-local boundary value problems associated with an elliptic operator. Ann. Scuola Norm. Sup. Pisa 22(3), 425-513 (1968)

8. Grubb, G.: Distributions and Operators, vol. 252 of Graduate Texts in Mathematics. Springer, New York (2009)

9. Kreĭn, M.G.: The theory of self-adjoint extensions of semi-bounded Hermitian transformations and its applications I. Rec. Math. [Mat. Sbornik] N.S. 20(62), 431-495 (1947)

10. Michelangeli, A., Ottolini, A.: On point interactions realised as Ter-Martirosyan-Skornyakov Hamiltonians. Rep. Math. Phys. 79, 215-260 (2017)

11. Schmüdgen, K.: Unbounded self-adjoint operators on Hilbert space. Graduate Texts in Mathematics, vol. 265. Springer, Dordrecht (2012)

12. Višik, M.I.: On general boundary problems for elliptic differential equations. Trudy Moskov. Mat. Obšč. 1, 187-246 (1952)

Publisher's Note Springer Nature remains neutral with regard to jurisdictional claims in published maps and institutional affiliations. 